\title{
Different Types of Hyers-Ulam-Rassias Stabilities for a Class of Integro-Differential Equations ${ }^{\dagger}$
}

\author{
L. P. Castro ${ }^{\text {, }}$ A. M. Simões ${ }^{a, b}$ \\ ${ }^{a}$ Center for Research and Development in Mathematics and Applications (CIDMA), \\ Department of Mathematics, University of Aveiro, Aveiro, Portugal \\ ${ }^{b}$ Center of Mathematics and Applications of University of Beira Interior (CMA-UBI), \\ Department of Mathematics, University of Beira Interior, Covilhã, Portugal
}

\begin{abstract}
We study different kinds of stabilities for a class of very general nonlinear integro-differential equations involving a function which depends on the solutions of the integro-differential equations and on an integral of Volterra type. In particular, we will introduce the notion of semi-Hyers-Ulam-Rassias stability, which is a type of stability somehow in-between the Hyers-Ulam and Hyers-Ulam-Rassias stabilities. This is considered in a framework of appropriate metric spaces in which sufficient conditions are obtained in view to guarantee Hyers-Ulam-Rassias, semi-Hyers-Ulam-Rassias and Hyers-Ulam stabilities for such a class of integro-differential equations. We will consider the different situations of having the integrals defined on finite and infinite intervals. Among the used techniques, we have fixed point arguments and generalizations of the Bielecki metric. Examples of the application of the proposed theory are included.
\end{abstract}

\section{Introduction}

The properties associated with the stability of functional, differential, integral and integro-differential equations have been studied in a quite extensive way during the last seven decades and have earned particular interest due to their great number of applications (see, e.g., [1, 3, 6, 8- $-11,13-17,19-25,28]$ and the references therein). This is the case of the stabilities of Hyers-Ulam and Hyers-Ulam-Rassias types. These stabilities were originated from a well-known question raised by S. M. Ulam at the University of Wisconsin in 1940: "When a solution of an equation differing slightly from a given one must be somehow near to the solution of the given equation?" A first partial answer to this question was given by D. H. Hyers, for Banach spaces, in the case of an additive Cauchy equation. This is why the obtained property is nowadays called the Hyers-Ulam stability. Afterwards, different generalizations of that initial answer of Hyers were obtained. Namely, there were extensions of that result within the framework of normed spaces by considering the possibility of using different exponents $p$ in some of the involved norms. Precisely in

2010 Mathematics Subject Classification. Primary 45M10; Secondary 34K20, 45J05

Keywords. Hyers-Ulam stability, semi-Hyers-Ulam-Rassias stability, Hyers-Ulam-Rassias stability, Banach fixed point theorem, integro-differential equation

Research supported by FCT-Portuguese Foundation for Science and Technology through the Center for Research and Development in Mathematics and Applications (CIDMA) of Universidade de Aveiro, within project UID/MAT/04106/2013, and by the Center of Mathematics and Applications of University of Beira Interior (CMA-UBI), within project UID/MAT/00212/2013.

${ }^{+}$Accepted author's manuscript (AAM) published in [Filomat Vol. 31, No. 17 (2017), 5379-5390] [DOI: 10.2298/FIL1717379C]. The final publication is available at http://journal.pmf.ni.ac.rs/filomat

Email addresses: castro@ua.pt (L. P. Castro), asimoes@ubi.pt (A. M. Simões) 
this sense, Aoki [2] obtained a result when $0<p<1$, Gajda [18] for $p>1$, and Rassias [27] for $p<0$. The interested reader can obtain a detailed description of these advances in [4].

Moreover, such stabilities were widely applied to different classes of functions and consequent functional equations. This development also gave rise to the introduction of a certain flexibility on the bounds used in the stability process. Namely, the possibility to use functions for that bounds (and not simply constants) was initially proposed by Th. M. Rassias, see [26], originating, therefore, a generalization of the initial concept, and making appear the so-called Hyers-Ulam-Rassias stability.

In this paper, which may be seen as a continuation of a somehow preliminary analysis presented in [12], we study different kinds of "Hyers-Ulam-Rassias stabilities" for the following class of nonlinear integro-differential equations,

$$
y^{\prime}(x)=f\left(x, y(x), \int_{a}^{x} k(x, \tau, y(\tau), y(\alpha(\tau))) d \tau\right), \quad y(a)=c \in \mathbb{R},
$$

with $y \in C^{1}([a, b])$, for $x \in[a, b]$ where, for starting, $a$ and $b$ are fixed real numbers, $f:[a, b] \times \mathbb{C} \times \mathbb{C} \rightarrow \mathbb{C}$ and $k:[a, b] \times[a, b] \times \mathbb{C} \times \mathbb{C} \rightarrow \mathbb{C}$ are continuous functions, and $\alpha:[a, b] \rightarrow[a, b]$ is a continuous delay function (i.e., fulfilling $\alpha(\tau) \leq \tau$ for all $\tau \in[a, b])$.

The formal definitions of the above mentioned stabilities are now introduced for our class of integrodifferential equations.

If for each function $y$ satisfying

$$
\left|y^{\prime}(x)-f\left(x, y(x), \int_{a}^{x} k(x, \tau, y(\tau), y(\alpha(\tau))) d \tau\right)\right| \leq \theta, \quad x \in[a, b],
$$

where $\theta \geq 0$, there is a solution $y_{0}$ of the integro-differential equation (1) and a constant $C>0$ independent of $y$ and $y_{0}$ such that

$$
\left|y(x)-y_{0}(x)\right| \leq C \theta,
$$

for all $x \in[a, b]$, then we say that the integro-differential equation (1) has the Hyers-Ulam stability.

If instead of $\theta$, in (2) and (3), we have a non-negative function $\sigma$ defined on $[a, b]$, then we say that the integro-differential equation (1) has the Hyers-Ulam-Rassias stability.

In this paper we introduce a new type of stability which we identify as in-between the two just mentioned stabilities of Hyers-Ulam-Rassias and Hyers-Ulam:

Definition 1.1. If for each function y satisfying

$$
\left|y^{\prime}(x)-f\left(x, y(x), \int_{a}^{x} k(x, \tau, y(\tau), y(\alpha(\tau))) d \tau\right)\right| \leq \theta, \quad x \in[a, b],
$$

where $\theta \geq 0$, there is a solution $y_{0}$ of the integro-differential equation (1) and a constant $C>0$ independent of $y$ and $y_{0}$ such that

$$
\left|y(x)-y_{0}(x)\right| \leq C \sigma(x), \quad x \in[a, b]
$$

for some non-negative function $\sigma$ defined on $[a, b]$, then we say that the integro-differential equation (1) has the semi-Hyers-Ulam-Rassias stability.

Some of the present techniques to study the stability of functional equations use a combination of fixed point results with a generalized metric in appropriate settings (cf. [5, 8- 11, 13, 14]). In view of this, and just for the sake of completeness, let us recall the definition of a generalized metric and the corresponding Banach Fixed Point Theorem.

Definition 1.2. We say that $d: X \times X \rightarrow[0,+\infty]$ is a generalized metric on $X$ if:

1. $d(x, y)=0$ if and only if $x=y$; 
2. $d(x, y)=d(y, x)$ for all $x, y \in X$;

3. $d(x, z) \leq d(x, y)+d(y, z)$ for all $x, y, z \in X$.

Theorem 1.3. Let $(X, d)$ be a generalized complete metric space and $T: X \rightarrow X$ a strictly contractive operator with a Lipschitz constant $L<1$. If there exists a nonnegative integer $k$ such that $d\left(T^{k+1} x, T^{k} x\right)<\infty$ for some $x \in X$, then the following three propositions hold true:

1. the sequence $\left(T^{n} x\right)_{n \in \mathbb{N}}$ converges to a fixed point $x^{*}$ of $T$;

2. $x^{*}$ is the unique fixed point of $T$ in $X^{*}=\left\{y \in X: d\left(T^{k} x, y\right)<\infty\right\}$;

3. if $y \in X^{*}$, then

$$
d\left(y, x^{*}\right) \leq \frac{1}{1-L} d(T y, y)
$$

\section{Hyers-Ulam-Rassias, semi-Hyers-Ulam-Rassias and Hyers-Ulam Stabilities in the Finite Interval Case}

We will present sufficient conditions for the Hyers-Ulam-Rassias, semi-Hyers-Ulam-Rassias and HyersUlam stabilities of the integro-differential equation (1), where $x \in[a, b]$, for some fixed real numbers $a$ and $b$.

In this section we will consider the space of continuously differentiable functions $C^{1}([a, b])$ on $[a, b]$ endowed with a Bielecki type metric

$$
d(u, v)=\sup _{x \in[a, b]} \frac{|u(x)-v(x)|}{\sigma(x)},
$$

where $\sigma$ is a non-decreasing continuous function $\sigma:[a, b] \rightarrow(0, \infty)$. We recall that $\left(C^{1}([a, b]), d\right)$ is a complete metric spaces (cf. [7, 29]).

Theorem 2.1. Let $\alpha:[a, b] \rightarrow[a, b]$ be a continuous delay function with $\alpha(t) \leq t$ for all $t \in[a, b]$ and $\sigma:[a, b] \rightarrow$ $(0, \infty)$ a non-decreasing continuous function. In addition, suppose that there is $\beta \in[0,1)$ such that

$$
\int_{a}^{x} \sigma(\tau) d \tau \leq \beta \sigma(x)
$$

for all $x \in[a, b]$. Moreover, suppose that $f:[a, b] \times \mathbb{C} \times \mathbb{C} \rightarrow \mathbb{C}$ is a continuous function satisfying the Lipschitz condition

$$
|f(x, u, g)-f(x, v, h)| \leq M(|u-v|+|g-h|)
$$

with $M>0$ and the kernel $k:[a, b] \times[a, b] \times \mathbb{C} \times \mathbb{C} \rightarrow \mathbb{C}$ is a continuous function satisfying the Lipschitz condition

$$
|k(x, t, u, w)-k(x, t, v, z)| \leq L|w-z|
$$

with $L>0$.

If $y \in C^{1}([a, b])$ is such that

$$
\left|y^{\prime}(x)-f\left(x, y(x), \int_{a}^{x} k(x, \tau, y(\tau), y(\alpha(\tau))) d \tau\right)\right| \leq \sigma(x), \quad x \in[a, b],
$$

and $M\left(\beta+L \beta^{2}\right)<1$, then there is a unique function $y_{0} \in C^{1}([a, b])$ such that

$$
y_{0}^{\prime}(x)=f\left(x, y_{0}(x), \int_{a}^{x} k\left(x, \tau, y_{0}(\tau), y_{0}(\alpha(\tau))\right) d \tau\right)
$$

and

$$
\left|y(x)-y_{0}(x)\right| \leq \frac{\beta \sigma(x)}{1-M\left(\beta+L \beta^{2}\right)}
$$

for all $x \in[a, b]$.

This means that under the above conditions, the integro-differential equation (1) has the Hyers-Ulam-Rassias stability. 
Proof. Using integration we realize that

$$
y^{\prime}(x)=f\left(x, y(x), \int_{a}^{x} k(x, \tau, y(\tau), y(\alpha(\tau))) d \tau\right)
$$

is equivalent to

$$
y(x)=c+\int_{a}^{x} f\left(s, y(s), \int_{a}^{s} k(s, \tau, y(\tau), y(\alpha(\tau))) d \tau\right) d s .
$$

So, we will consider the operator $T: C^{1}([a, b]) \rightarrow C^{1}([a, b])$, defined by

$$
(T u)(x)=c+\int_{a}^{x} f\left(s, u(s), \int_{a}^{s} k(s, \tau, u(\tau), u(\alpha(\tau))) d \tau\right) d s,
$$

for all $x \in[a, b]$ and $u \in C^{1}([a, b])$.

Note that for any continuous function $u, T u$ is also continuous. Indeed,

$$
\begin{aligned}
\left|(T u)(x)-(T u)\left(x_{0}\right)\right| & =\mid \int_{a}^{x} f\left(s, u(s), \int_{a}^{s} k(s, \tau, u(\tau), u(\alpha(\tau))) d \tau\right) d s \\
& \quad-\int_{a}^{x_{0}} f\left(s, u(s), \int_{a}^{s} k(s, \tau, u(\tau), u(\alpha(\tau))) d \tau\right) d s \mid \\
& =\left|\int_{x}^{x_{0}} f\left(s, u(s), \int_{a}^{s} k(s, \tau, u(\tau), u(\alpha(\tau))) d \tau\right) d s\right| \rightarrow 0
\end{aligned}
$$

when $x \rightarrow x_{0}$.

Under the present conditions, we will deduce that the operator $T$ is strictly contractive with respect to the metric (7). Indeed, for all $u, v \in C^{1}([a, b])$, we have,

$$
\begin{aligned}
d(T u, T v)= & \sup _{x \in[a, b]} \frac{|(T u)(x)-(T v)(x)|}{\sigma(x)} \\
\leq & M \sup _{x \in[a, b]} \frac{1}{\sigma(x)} \int_{a}^{x}|u(s)-v(s)| d s \\
& \quad+M \sup _{x \in[a, b]} \frac{1}{\sigma(x)} \int_{a}^{x} \int_{a}^{s}|k(s, \tau, u(\tau), u(\alpha(\tau)))-k(s, \tau, v(\tau), v(\alpha(\tau)))| d \tau d s \\
\leq & M \sup _{x \in[a, b]} \frac{1}{\sigma(x)} \int_{a}^{x}|u(s)-v(s)| d s+M L \sup _{x \in[a, b]} \frac{1}{\sigma(x)} \int_{a}^{x} \int_{a}^{s}|u(\alpha(\tau))-v(\alpha(\tau))| d \tau d s \\
= & M \sup _{x \in[a, b]} \frac{1}{\sigma(x)} \int_{a}^{x} \sigma(s) \frac{|u(s)-v(s)|}{\sigma(s)} d s \\
& \quad+M L \sup _{x \in[a, b]} \frac{1}{\sigma(x)} \int_{a}^{x} \int_{a}^{s} \sigma(\tau) \frac{|u(\alpha(\tau))-v(\alpha(\tau))|}{\sigma(\tau)} d \tau d s \\
\leq & M \sup _{s \in[a, b]} \frac{|u(s)-v(s)|}{\sigma(s)} \sup _{x \in[a, b]} \frac{1}{\sigma(x)} \int_{a}^{x} \sigma(s) d s \\
& \quad+M L \sup _{\tau \in[a, b]} \frac{|u(\tau)-v(\tau)|}{\sigma(\tau)} \sup _{x \in[a, b]} \frac{1}{\sigma(x)} \int_{a}^{x} \int_{a}^{s} \sigma(\tau) d \tau d s \\
\leq & M d(u, v) \beta+M L d(u, v) \sup _{x \in[a, b]} \frac{\beta^{2} \sigma(x)}{\sigma(x)} \\
= & M\left(\beta+L \beta^{2}\right) d(u, v) .
\end{aligned}
$$


Due to the fact that $M\left(\beta+L \beta^{2}\right)<1$ it follows that $T$ is strictly contractive. Thus, we can apply the above mentioned Banach Fixed Point Theorem which allows us to ensure that we have the Hyers-Ulam-Rassias stability for the integro-differential equation.

Indeed, from (11), we have

$$
-\sigma(x) \leq y^{\prime}(x)-f\left(x, y(x), \int_{a}^{x} k(x, \tau, y(\tau), y(\alpha(\tau))) d \tau\right) \leq \sigma(x), \quad x \in[a, b]
$$

and so, using integration in (19), we obtain

$$
\left|y(x)-c-\int_{a}^{x} f\left(s, y(s), \int_{a}^{s} k(s, \tau, y(\tau), y(\alpha(\tau))) d \tau\right) d s\right| \leq \int_{a}^{x} \sigma(\tau) d \tau, \quad x \in[a, b] .
$$

Therefore, having in mind (16) and (8), we conclude that

$$
|y(x)-T y(x)| \leq \int_{a}^{x} \sigma(\tau) d \tau \leq \beta \sigma(x), \quad x \in[a, b] .
$$

Consequently, (13) follows directly from the definition of the metric $d,(6)$ and (21).

Now, we will consider the semi-Hyers-Ulam-Rassias and the Hyers-Ulam stabilities of the integrodifferential equation (1).

Theorem 2.2. Let $\alpha:[a, b] \rightarrow[a, b]$ be a continuous delay function with $\alpha(t) \leq t$ for all $t \in[a, b]$ and $\sigma:[a, b] \rightarrow$ $(0, \infty)$ a non-decreasing continuous function. In addition, suppose that there is $\beta \in[0,1)$ such that

$$
\int_{a}^{x} \sigma(\tau) d \tau \leq \beta \sigma(x)
$$

for all $x \in[a, b]$. Moreover, suppose that $f:[a, b] \times \mathbb{C} \times \mathbb{C} \rightarrow \mathbb{C}$ is a continuous function satisfying the Lipschitz condition

$$
|f(x, u, g)-f(x, v, h)| \leq M(|u-v|+|g-h|)
$$

with $M>0$ and $k:[a, b] \times[a, b] \times \mathbb{C} \times \mathbb{C} \rightarrow \mathbb{C}$ is a continuous kernel function satisfying the Lipschitz condition

$$
|k(x, t, u, w)-k(x, t, v, z)| \leq L|w-z|
$$

with $L>0$.

If $y \in C^{1}([a, b])$ is such that

$$
\left|y^{\prime}(x)-f\left(x, y(x), \int_{a}^{x} k(x, \tau, y(\tau), y(\alpha(\tau))) d \tau\right)\right| \leq \theta, \quad x \in[a, b],
$$

where $\theta>0$ and $M\left(\beta+L \beta^{2}\right)<1$, then there is a unique function $y_{0} \in C^{1}([a, b])$ such that

$$
y_{0}^{\prime}(x)=f\left(x, y_{0}(x), \int_{a}^{x} k\left(x, t, y_{0}(t), y_{0}(\alpha(t))\right) d t\right)
$$

and

$$
\left|y(x)-y_{0}(x)\right| \leq \frac{(b-a) \theta}{\left[1-M\left(\beta+L \beta^{2}\right)\right] \sigma(a)} \sigma(x)
$$

for all $x \in[a, b]$.

This means that under the above conditions, the integro-differential equation (1) has the semi-Hyers-Ulam-Rassias stability. 
Proof. The first part of the proof of this result follows the same steps as in the proof of Theorem 2.2. The main argument is that we maintain $\sigma$ as general as before in its role in the metric $d$, doing exactly the same reasoning as in (14)-(18), and just take it as a constant in all the remaining places.

Therefore, we consider again the operator $T: C^{1}([a, b]) \rightarrow C^{1}([a, b])$, defined by

$$
(T u)(x)=c+\int_{a}^{x} f\left(s, u(s), \int_{a}^{s} k(s, \tau, u(\tau), u(\alpha(\tau))) d \tau\right) d s,
$$

for all $x \in[a, b]$ and $u \in C^{1}([a, b])$, and by using the same reasoning as in (17)-(18) we conclude that $T$ is strictly contractive with respect to the metric (7), due to the fact that $M\left(\beta+L \beta^{2}\right)<1$. Thus, we can again apply the Banach Fixed Point Theorem, which guarantees us that

$$
d\left(y, y_{0}\right) \leq \frac{1}{1-M\left(\beta+L \beta^{2}\right)} d(T y, y) .
$$

Now, due to (25), instead of (19) we have

$$
-\theta \leq y^{\prime}(x)-f\left(x, y(x), \int_{a}^{x} k(x, \tau, y(\tau), y(\alpha(\tau))) d \tau\right) \leq \theta, \quad x \in[a, b],
$$

and so, using integration in (30), we obtain

$$
\left|y(x)-c-\int_{a}^{x} f\left(s, y(s), \int_{a}^{s} k(s, \tau, y(\tau), y(\alpha(\tau))) d \tau\right) d s\right| \leq \int_{a}^{x} \theta d \tau, \quad x \in[a, b]
$$

and conclude that

$$
|y(x)-T y(x)| \leq(b-a) \theta, \quad x \in[a, b] .
$$

Using now this last information in (29), and having in mind (7) and the circumstance that $\sigma$ is a positive non-decreasing function, it follows

$$
\begin{aligned}
\sup _{x \in[a, b]} \frac{\left|y(x)-y_{0}(x)\right|}{\sigma(x)} & \leq \frac{1}{1-M\left(\beta+L \beta^{2}\right)} \sup _{x \in[a, b]} \frac{(b-a) \theta}{\sigma(x)} \\
& \leq \frac{1}{1-M\left(\beta+L \beta^{2}\right)} \frac{(b-a) \theta}{\sigma(a)}
\end{aligned}
$$

Consequently, (27) follows directly from (33) and this leads us to the semi-Hyers-Ulam-Rassias stability of the integro-differential equation under study.

Still having in mind that $\sigma$ is a positive non-decreasing function, and considering an obvious upper bound in (27), we directly obtain from the last result the following Hyers-Ulam stability of the integrodifferential equation (1).

Corollary 2.3. Let $\alpha:[a, b] \rightarrow[a, b]$ be a continuous delay function with $\alpha(t) \leq t$ for all $t \in[a, b]$ and $\sigma:[a, b] \rightarrow$ $(0, \infty)$ a non-decreasing continuous function. In addition, suppose that there is $\beta \in[0,1)$ such that

$$
\int_{a}^{x} \sigma(\tau) d \tau \leq \beta \sigma(x)
$$

for all $x \in[a, b]$. Moreover, suppose that $f:[a, b] \times \mathbb{C} \times \mathbb{C} \rightarrow \mathbb{C}$ is a continuous function satisfying the Lipschitz condition

$$
|f(x, u, g)-f(x, v, h)| \leq M(|u-v|+|g-h|)
$$

with $M>0$ and $k:[a, b] \times[a, b] \times \mathbb{C} \times \mathbb{C} \rightarrow \mathbb{C}$ is a continuous kernel function satisfying the Lipschitz condition

$$
|k(x, t, u, w)-k(x, t, v, z)| \leq L|w-z|
$$


with $L>0$.

If $y \in C^{1}([a, b])$ is such that

$$
\left|y^{\prime}(x)-f\left(x, y(x), \int_{a}^{x} k(x, \tau, y(\tau), y(\alpha(\tau))) d \tau\right)\right| \leq \theta, \quad x \in[a, b],
$$

where $\theta>0$ and $M\left(\beta+L \beta^{2}\right)<1$, then there is a unique function $y_{0} \in C^{1}([a, b])$ such that

$$
y_{0}^{\prime}(x)=f\left(x, y_{0}(x), \int_{a}^{x} k\left(x, t, y_{0}(t), y_{0}(\alpha(t))\right) d t\right)
$$

and

$$
\left|y(x)-y_{0}(x)\right| \leq \frac{(b-a) \theta \sigma(b)}{\left[1-M\left(\beta+L \beta^{2}\right)\right] \sigma(a)}, \quad x \in[a, b] .
$$

This means that under the above conditions, the integro-differential equation (1) has the Hyers-Ulam stability.

\section{Hyers-Ulam-Rassias Stability in the Infinite Interval Case}

In this section, we analyse the Hyers-Ulam-Rassias stability of the integro-differential equation (1) but, instead of considering a finite interval $[a, b]$ (with $a, b \in \mathbb{R}$ ), we will consider the infinite interval $[a, \infty)$, for some fixed $a \in \mathbb{R}$.

Thus, we will now be dealing with the integro-differential equation

$$
y^{\prime}(x)=f\left(x, y(x), \int_{a}^{x} k(x, \tau, y(\tau), y(\alpha(\tau))) d \tau\right), \quad y(a)=c \in \mathbb{R},
$$

with $y \in C^{1}([a, \infty)), x \in[a, \infty)$ where $a$ is a fixed real number, $f:[a, \infty) \times \mathbb{C} \times \mathbb{C} \rightarrow \mathbb{C}$ and $k:[a, \infty) \times[a, \infty) \times$ $\mathbb{C} \times \mathbb{C} \rightarrow \mathbb{C}$ are continuous functions, and $\alpha:[a, \infty) \rightarrow[a, \infty)$ is a continuous delay function which therefore fulfills $\alpha(\tau) \leq \tau$ for all $\tau \in[a, \infty)$.

The main strategy will be based on a recurrence procedure due to the already obtained result for the corresponding finite interval case.

Let us now consider a fixed non-decreasing continuous function $\sigma:[a, \infty) \rightarrow(\varepsilon, \omega)$, for some $\varepsilon, \omega>0$ and the space $C_{b}^{1}([a, \infty))$ of bounded differentiable functions endowed with the metric

$$
d_{b}(u, v)=\sup _{x \in[a, \infty)} \frac{|u(x)-v(x)|}{\sigma(x)} .
$$

Theorem 3.1. Let $\alpha:[a, \infty) \rightarrow[a, \infty)$ be a continuous delay function with $\alpha(t) \leq t$, for all $t \in[a, \infty)$, and $\sigma:[a, \infty) \rightarrow(\varepsilon, \omega)$, for some $\varepsilon, \omega>0$, a non-decreasing continuous function. Suppose that there is $\beta \in[0,1)$ such that

$$
\int_{a}^{x} \sigma(\tau) d \tau \leq \beta \sigma(x)
$$

for all $x \in[a, \infty)$. Moreover, suppose that $f:[a, \infty) \times \mathbb{C} \times \mathbb{C} \rightarrow \mathbb{C}$ is a continuous function satisfying the Lipschitz condition

$$
|f(x, u, g)-f(x, v, h)| \leq M(|u-v|+|g-h|)
$$

with $M>0$ and the kernel $k:[a, \infty) \times[a, \infty) \times \mathbb{C} \times \mathbb{C} \rightarrow \mathbb{C}$ is a continuous function so that $\int_{a}^{x} k(x, \tau, z(\tau), z(\alpha(\tau))) d \tau$ is a bounded continuous function for any bounded continuous function $z$. In addition, suppose that $k$ satisfies the Lipschitz condition

$$
|k(x, t, u, w)-k(x, t, v, z)| \leq L|w-z|
$$


with $L>0$.

If $y \in C_{b}^{1}([a, \infty))$ is such that

$$
\left|y^{\prime}(x)-f\left(x, y(x), \int_{a}^{x} k(x, \tau, y(\tau), y(\alpha(\tau))) d \tau\right)\right| \leq \sigma(x), \quad x \in[a, \infty),
$$

and $M\left(\beta+L \beta^{2}\right)<1$, then there is a unique function $y_{0} \in C_{b}^{1}([a, b])$ such that

$$
y_{0}^{\prime}(x)=f\left(x, y_{0}(x), \int_{a}^{x} k\left(x, \tau, y_{0}(\tau), y_{0}(\alpha(\tau))\right) d \tau\right)
$$

and

$$
\left|y(x)-y_{0}(x)\right| \leq \frac{\beta \sigma(x)}{1-M\left(\beta+L \beta^{2}\right)}
$$

for all $x \in[a, \infty)$.

This means that under the above conditions, the integro-differential equation (40) has the Hyers-Ulam-Rassias stability.

Proof. For any $n \in \mathbb{N}$, we will define $I_{n}=[a, a+n]$. By Theorem 2.1, there exists a unique bounded differentiable function $y_{0, n}: I_{n} \rightarrow \mathbb{C}$ such that

$$
y_{0, n}(x)=c+\int_{a}^{x} f\left(s, y_{0, n}(s), \int_{a}^{s} k\left(s, \tau, y_{0, n}(\tau), y_{0, n}(\alpha(\tau))\right) d \tau\right) d s
$$

and

$$
\left|y(x)-y_{0, n}(x)\right| \leq \frac{\beta \sigma(x)}{1-M\left(\beta+L \beta^{2}\right)}
$$

for all $x \in I_{n}$. The uniqueness of $y_{0, n}$ implies that if $x \in I_{n}$ then

$$
y_{0, n}(x)=y_{0, n+1}(x)=y_{0, n+2}(x)=\cdots .
$$

For any $x \in[a, \infty)$, let us define $n(x) \in \mathbb{N}$ as $n(x)=\min \left\{n \in \mathbb{N}: x \in I_{n}\right\}$. We also define a function $y_{0}:[a, \infty) \rightarrow \mathbb{C}$ by

$$
y_{0}(x)=y_{0, n(x)}(x)
$$

For any $x_{1} \in[a, \infty)$, let $n_{1}=n\left(x_{1}\right)$. Then $x_{1} \in \operatorname{Int} I_{n_{1}+1}$ and there exists an $\epsilon>0$ such that $y_{0}(x)=y_{0, n_{1}+1}(x)$ for all $x \in\left(x_{1}-\epsilon, x_{1}+\epsilon\right)$. By Theorem 2.1, $y_{0, n_{1}+1}$ is continuous at $x_{1}$, and so it is $y_{0}$.

Now, we will prove that $y_{0}$ satisfies

$$
y_{0}(x)=c+\int_{a}^{x} f\left(s, y_{0}(s), \int_{a}^{s} k\left(s, \tau, y_{0}(\tau), y_{0}(\alpha(\tau))\right) d \tau\right) d s
$$

and (47). For an arbitrary $x \in[a, \infty)$ we choose $n(x)$ such that $x \in I_{n(x)}$. By (48) and (51), we have

$$
\begin{aligned}
y_{0}(x)=y_{0, n(x)}(x) & =c+\int_{a}^{x} f\left(s, y_{0, n(x)}(s), \int_{a}^{s} k\left(s, \tau, y_{0, n(x)}(\tau), y_{0, n(x)}(\alpha(\tau))\right) d \tau\right) d s \\
& =c+\int_{a}^{x} f\left(s, y_{0}(s), \int_{a}^{s} k\left(s, \tau, y_{0}(\tau), y_{0}(\alpha(\tau))\right) d \tau\right) d s .
\end{aligned}
$$

Note that $n(\tau) \leq n(x)$, for any $\tau \in I_{n(x)}$, and it follows from (50) that $y_{0}(\tau)=y_{0, n(\tau)}(\tau)=y_{0, n(x)}(\tau)$, so, the last equality in (53) holds true.

To prove (47), by (51) and (49), we have that for all $x \in[a, \infty)$,

$$
\left|y(x)-y_{0}(x)\right|=\left|y(x)-y_{0, n(x)}(x)\right| \leq \frac{\beta \sigma(x)}{1-M\left(\beta+L \beta^{2}\right)} .
$$


Finally, we will prove the uniqueness of $y_{0}$. Let us consider another bounded differentiable function $y_{1}$ which satisfies (46) and (47), for all $x \in[a, \infty)$. By the uniqueness of the solution on $I_{n(x)}$ for any $n(x) \in \mathbb{N}$ we have that $y_{\left.\right|_{I_{n(x)}}}=y_{0, n(x)}$ and $y_{1_{\mid I_{n(x)}}}$ satisfies (46) and (47) for all $x \in I_{n(x)}$, and so

$$
y_{0}(x)=\left.y_{0}\right|_{I_{n(x)}}(x)=\left.y_{1}\right|_{I_{n(x)}}(x)=y_{1}(x) \text {. }
$$

The theorem is proved.

We would like to point out that with the necessary adaptations, Theorem 3.1 also holds true for infinite intervals of the type $(-\infty, b]$, with $b \in \mathbb{R}$, as well as for $(-\infty, \infty)$.

\section{Illustrative Examples}

In this section we will present three examples simply to illustrate that the conditions of the above results are possible to attain.

For differentiable functions $y:[0,1] \rightarrow \mathbb{R}$, let us start be considering the integro-differential equation

$$
y^{\prime}(x)=(-2 x-4) e^{x / 2}+5 y(x)+e^{x / 2} \int_{0}^{x}((\tau-x) y(\alpha(\tau))) d \tau, \quad x \in[0,1],
$$

as well as the continuous function $\sigma:[0,1] \rightarrow(0, \infty)$ defined by $\sigma(x)=1.1 e^{10 x}$ and the continuous delay function $\alpha:[0,1] \rightarrow[0,1 / 2]$ given by $\alpha(x)=x / 2$.

We have all the conditions of Theorem 2.2 being satisfied. In fact, such $\alpha:[0,1] \rightarrow[0,1 / 2]$ defined by $\alpha(x)=x / 2$ is a continuous function, and obviously $\alpha(x) \leq x$. Moreover, for $\beta=1 / 10$ we have that $\sigma:[0,1] \rightarrow(0, \infty)$ defined by $\sigma(x)=1.1 e^{10 x}$ is a continuous function fulfilling

$$
\int_{0}^{x} 1.1 e^{10 \tau} d \tau \leq \beta 1.1 e^{10 x}=\frac{1}{10} \sigma(x), \quad x \in[0,1]
$$

$f:[0,1] \times \mathbb{C} \times \mathbb{C} \rightarrow \mathbb{C}$ defined by $f(x, y(x), g(x))=(-2 x-4) e^{x / 2}+5 y(x)+e^{x / 2} g(x)$ is a continuous function which fulfills

$$
|f(x, u(x), g(x))-f(x, v(x), h(x))| \leq 5(|u(x)-v(x)|+|g(x)-h(x)|), \quad x \in[0,1]
$$

(and so the previous constant $M$ is here taking the value 5); the kernel $k:[0,1] \times[0,1] \times \mathbb{C} \rightarrow \mathbb{C}$ defined by $k(x, \tau, y(\tau), y(\alpha(\tau)))=(\tau-x) y(\alpha(\tau))$ is a continuous function which fulfils the condition

$$
|k(x, \tau, u(\tau), u(\alpha(\tau)))-k(x, \tau, v(\tau), v(\alpha(\tau)))| \leq\left|u\left(\frac{\tau}{2}\right)-v\left(\frac{\tau}{2}\right)\right|, \quad \tau \in[0, x], \quad x \in[0,1]
$$

(where we may identify 1 as the constant previously denoted by $L$ ). Thus, $M\left(\beta+L \beta^{2}\right)=11 / 20<1$.

If we choose $y(x)=100 e^{x} / 99$, it follows

$$
\left|y^{\prime}(x)-f\left(x, y(x), \int_{a}^{x} k(x, \tau, y(\tau), y(\alpha(\tau))) d \tau\right)\right|=\left|\left(-\frac{2}{99} x-\frac{4}{99}\right) e^{x / 2}\right| \leq \theta:=0.1, \quad x \in[0,1] .
$$

Therefore, from Theorem 2.2, we have the semi-Hyers-Ulam-Rassias stability of the integro-differential equation (56), and from Corollary 2.3 we have the consequent Hyers-Ulam stability. In particular, having in mind the exact solution $y_{0}(x)=e^{x}$ of (56), it follows that

$$
\left|y(x)-y_{0}(x)\right|=\left|\frac{100}{99} e^{x}-e^{x}\right| \leq \frac{(b-a) \theta}{\left[1-M\left(\beta+L \beta^{2}\right)\right] \sigma(a)} \sigma(x)=\frac{2}{9} e^{10 x}, \quad x \in[0,1],
$$

Let us now turn to a second example in which the Hyers-Ulam-Rassias stability is illustrated. For a differentiable function $y:\left[0, \frac{\pi}{2}\right] \rightarrow \mathbb{R}$, we shall consider the integro-differential equation

$$
y^{\prime}(x)=\frac{y(x)+x+1}{1+2 x}-\frac{1}{1+2 x} \int_{0}^{x}((x+\tau+1) y(\tau)) d \tau, \quad x \in\left[0, \frac{\pi}{2}\right],
$$


as well as the continuous function $\sigma:\left[0, \frac{\pi}{2}\right] \rightarrow(0, \infty)$ defined by $\sigma(x)=e^{5 x}$ and take simply the identity function for what was previously denoted by $\alpha:\left[0, \frac{\pi}{2}\right] \rightarrow\left[0, \frac{\pi}{2}\right]$.

We realize that all the conditions of Theorem 2.1 are here satisfied. In fact, $\alpha(x)=x$ is obviously continuous and satisfies $\alpha(x) \leq x$; for e.g. $\beta=1 / 3, \sigma:\left[0, \frac{\pi}{2}\right] \rightarrow[0, \infty)$ defined by $\sigma(x)=e^{5 x}$ is a continuous function which fulfills

$$
\int_{0}^{x} e^{5 \tau} d \tau \leq \frac{1}{3} e^{5 x}=\beta \sigma(x), \quad x \in\left[0, \frac{\pi}{2}\right]
$$

$f:\left[0, \frac{\pi}{2}\right] \times \mathbb{C} \times \mathbb{C} \rightarrow \mathbb{C}$ defined by $f(x, y(x), g(x))=\frac{y(x)+x+1}{1+2 x}-\frac{1}{1+2 x} g(x)$ is a continuous function which fulfills

$$
|f(x, u(x), g(x))-f(x, v(x), h(x))| \leq|u(x)-v(x)|+|g(x)-h(x)|, \quad x \in\left[0, \frac{\pi}{2}\right]
$$

(where we may identify $M=1$ ); the kernel $k:\left[0, \frac{\pi}{2}\right] \times\left[0, \frac{\pi}{2}\right] \times \mathbb{C} \rightarrow \mathbb{C}$ defined by $k(x, \tau, y(\tau), y(\alpha(\tau)))=$ $(x+\tau+1) y(\tau)$ is a continuous function which fulfils the condition

$$
|k(x, \tau, u(\tau), u(\alpha(\tau)))-k(x, \tau, v(\tau), v(\alpha(\tau)))| \leq(\pi+1)|u(\tau)-v(\tau)|, \quad \tau \in[0, x], \quad x \in\left[0, \frac{\pi}{2}\right],
$$

where we make the identification $L=\pi+1$. Thus, $M\left(\beta+L \beta^{2}\right)=(\pi+4) / 9<1$. Moreover, if we choose $y(x)=\frac{\sin (x)}{0.99}$, it follows,

$$
\left|y^{\prime}(x)-f\left(x, y(x), \int_{a}^{x} k(x, \tau, y(\tau), y(\alpha(\tau))) d \tau\right)\right|=\left|\frac{0.01 x+0.01}{0.99+1.98 x}\right| \leq \sigma(x), \quad x \in\left[0, \frac{\pi}{2}\right] .
$$

Therefore, the integro-differential equation (62) exhibits the Hyers-Ulam-Rassias stability.

In particular, by using the exact solution $y_{0}(x)=\sin (x)$, we realize that

$$
\left|y(x)-y_{0}(x)\right|=\left|\frac{\sin (x)}{0.99}-\sin (x)\right| \leq \frac{\beta \sigma(x)}{1-M\left(\beta+L \beta^{2}\right)}=\frac{3}{5-\pi} e^{5 x}, \quad x \in\left[0, \frac{\pi}{2}\right] .
$$

We will consider a third and final example. For a differentiable function $y:\left[0, \frac{2}{5}\right] \rightarrow \mathbb{R}$, let us consider the integro-differential equation

$$
y^{\prime}(x)=1+2 x-y(x)+\int_{0}^{x}\left(x(1+2 x) y(\tau) e^{\tau(x-\tau)}\right) d \tau, \quad x \in\left[0, \frac{2}{5}\right],
$$

as well as the continuous function $\sigma:\left[0, \frac{2}{5}\right] \rightarrow(0, \infty)$ defined by $\sigma(x)=3 e^{x}$ and take the function previously denoted by $\alpha:\left[0, \frac{2}{5}\right] \rightarrow\left[0, \frac{2}{5}\right]$ to be the identity function.

For $\beta=1 / 2$ we realize that $\sigma(x)=3 e^{x}$ fulfills

$$
\int_{0}^{x} 3 e^{\tau} d \tau \leq \frac{3}{2} e^{x}=\beta \sigma(x), \quad x \in\left[0, \frac{2}{5}\right] .
$$

Additionally, $f:\left[0, \frac{2}{5}\right] \times \mathbb{C} \times \mathbb{C} \rightarrow \mathbb{C}$ defined by $f(x, y(x), g(x))=1+2 x-y(x)+g(x)$ is a continuous function which satisfies

$$
|f(x, u(x), g(x))-f(x, v(x), h(x))| \leq|u(x)-v(x)|+|g(x)-h(x)|, \quad x \in\left[0, \frac{2}{5}\right],
$$

and so we may take the constant $M$ considered in Theorem 2.1 to be equal to 1 . As about the kernel $k:\left[0, \frac{2}{5}\right] \times\left[0, \frac{2}{5}\right] \times \mathbb{C} \rightarrow \mathbb{C}$, we identify it as $k(x, \tau, y(\tau), y(\alpha(\tau)))=x(1+2 x) y(\tau) e^{\tau(x-\tau)}$ and so it is a continuous function which fulfils the condition

$$
|k(x, \tau, u(\tau), u(\alpha(\tau)))-k(x, \tau, v(\tau), v(\alpha(\tau)))| \leq \frac{18}{25} e^{\frac{1}{25}}|u(\tau)-v(\tau)|, \quad \tau \in[0, x], \quad x \in\left[0, \frac{2}{5}\right],
$$


where we are using the constant $L=\frac{18}{25} e^{\frac{1}{25}}$. Consequently, we have $M\left(\beta+L \beta^{2}\right)=\frac{1}{2}+\frac{9}{50} e^{1 / 25}<1$.

Moreover, taking $y(x)=\frac{e^{x^{2}}}{0.3}$, it follows,

$$
\left|y^{\prime}(x)-f\left(x, y(x), \int_{a}^{x} k(x, \tau, y(\tau), y(\alpha(\tau))) d \tau\right)\right|=\left|\frac{7}{3}+\frac{14}{3} x\right| \leq \sigma(x), \quad x \in\left[0, \frac{2}{5}\right] .
$$

As a consequence, from Theorem 2.1, we have that the integro-differential equation (68) has the HyersUlam-Rassias stability.

Moreover, by using the exact solution $y_{0}(x)=e^{x^{2}}$, we have that

$$
\left|y(x)-y_{0}(x)\right|=\left|\frac{e^{x^{2}}}{0.3}-e^{x^{2}}\right| \leq \frac{3 e^{x}}{1-\frac{9}{25} e^{1 / 25}}=\frac{\beta \sigma(x)}{1-M\left(\beta+L \beta^{2}\right)}, \quad x \in\left[0, \frac{2}{5}\right] .
$$

Still within this last example associated with the integro-differential equation (68), and using the same $\beta, M$ and $L$ (and so still having $M\left(\beta+L \beta^{2}\right)=\frac{1}{2}+\frac{9}{50} e^{1 / 25}<1$ ), we will now exemplify that we may also have different possibilities for the choice of $\sigma$. Indeed, let us now consider for $\sigma$ the continuous function $\sigma:\left[0, \frac{2}{5}\right] \rightarrow[0, \infty)$ defined by $\sigma(x)=0.011+0.019 x$. If this is the case, instead of (69), we have now

$$
\int_{0}^{x} 0.011+0.019 \tau d \tau \leq \frac{1}{2}(0.011+0.019 x)=\beta \sigma(x), \quad x \in\left[0, \frac{2}{5}\right] .
$$

Considering now $y(x)=\frac{e^{x^{2}}}{0.99}$, it follows

$$
\left|y^{\prime}(x)-f\left(x, y(x), \int_{a}^{x} k(x, \tau, y(\tau), y(\alpha(\tau))) d \tau\right)\right|=\left|\frac{1}{99}+\frac{2}{99} x\right| \leq \sigma(x),
$$

for all $x \in\left[0, \frac{2}{5}\right]$, and so we have a different way to illustrate the Hyers-Ulam-Rassias stability of the integro-differential equation (68).
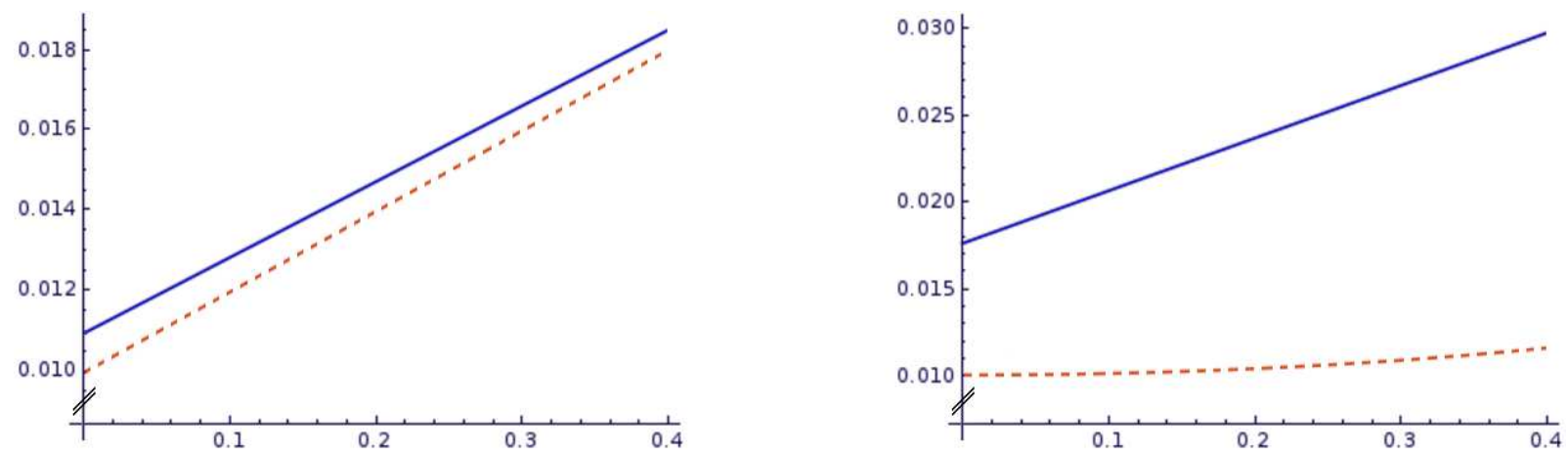

Figure 1: On the left, denoted by a continuous line we have $\sigma(x)=0.011+0.019 x$, and denoted by a dotted line $z(x)=\left|\frac{1}{99}+\frac{2}{99} x\right|$, allowing a comparison between the two functions which appear in the inequality $\left[75\right.$; on the right, $\omega(x)=\frac{\beta \sigma(x)}{1-M\left(\beta+L \beta^{2}\right)}=\frac{0.011+0.019 x}{1-\frac{9}{25} e^{1 / 25}}$ is denoted by a continuous line, and $w(x)=\left|\frac{e^{x^{2}}}{0.99}-e^{x^{2}}\right|$ is denoted by a dotted line, allowing a comparison between the two functions involved in [76.

Moreover, by using the exact solution $y_{0}(x)=e^{x^{2}}$, it follows

$$
\left|y(x)-y_{0}(x)\right|=\left|\frac{e^{x^{2}}}{0.99}-e^{x^{2}}\right| \leq \frac{0.011+0.019 x}{1-\frac{9}{25} e^{1 / 25}}=\frac{\beta \sigma(x)}{1-M\left(\beta+L \beta^{2}\right)}, \quad x \in\left[0, \frac{2}{5}\right] .
$$

To illustrate the last case, when considering $y(x)=\frac{e^{x^{2}}}{0.99}, \sigma(x)=0.011+0.019 x, y_{0}(x)=e^{x^{2}}, M=1, L=\frac{18}{25} e^{\frac{1}{25}}$ and $\beta=0.5$, Figure 1 presents the graphs of the functions appearing in (75) and (76). 


\section{Acknowledgment}

The authors are grateful to the referee's valuable suggestions and insightful comments.

\section{References}

[1] H. H. Alsulami, S. Gülyaz, E. Karapinar, I. M. Erhan, An Ulam stability result on quasi-b-metric-like spaces, Open Math. 14 (2016) 1087-1103.

[2] T. Aoki, On the stability of the linear transformation in Banach spaces, J. Math. Soc. Japan 2 (1950) 64-66.

[3] A. Bahyrycz, J. Brzdęk, E. Jablońska, R. Malejki, Ulam's stability of a generalization of the Fréchet functional equation, J. Math. Anal. Appl. 442(2) (2016) 537-553.

[4] N. B.-Belluot, J. Brzdęk, K. Ciepliński, On some recent developments in Ulam's type stability, Abstr. Appl. Anal. 2012 (2012) $41 \mathrm{pp}$.

[5] J. Brzdęk, L. Cădariu, K. Ciepliński, Fixed point theory and the Ulam stability, J. Funct. Spaces 2014 Article ID 829419 (2014) $16 \mathrm{pp}$.

[6] T. A. Burton, Volterra Integral and Differential Equations (2nd ed.), Mathematics in Science and Engineering 202, Elsevier Science, Amsterdam, 2005.

[7] L. Cădariu, L. Găvruța, P. Găvruça, Weighted space method for the stability of some nonlinear equations, Appl. Anal. Discrete Math. 6(1) (2012) 126-139.

[8] L. P. Castro, R. C. Guerra, Hyers-Ulam-Rassias stability of Volterra integral equations within weighted spaces, Lib. Math. (N.S.) 33(2) (2013) 21-35.

[9] L. P. Castro, A. Ramos, Hyers-Ulam and Hyers-Ulam-Rassias stability of Volterra integral equations with a delay, in "Integral Methods in Science and Engineering. Volume 1: Analytic Methods", C. Constanda (ed.) et al., Birkhäuser, Basel, (2010) 85-94.

[10] L. P. Castro, A. Ramos, Hyers-Ulam-Rassias stability for a class of nonlinear Volterra integral equations, Banach J. Math. Anal. 3(1) (2009) 36-43.

[11] L. P. Castro, A. Ramos, Hyers-Ulam stability for a class of Fredholm integral equations, in "Mathematical Problems in Engineering Aerospace and Sciences ICNPAA 2010", Proceedings of the 8th International Conference of Mathematical Problems in Engineering, Aerospace and Science (2011) 171-176.

[12] L. P. Castro, A. M. Simões, Hyers-Ulam and Hyers-Ulam-Rassias stability for a class of integro-differential equations, in "Mathematical Methods in Engineering: Theoretical Aspects", K. Tas, D. Baleanu and J.A. Tenreiro Machado (edts.), Springer, to appear.

[13] L. P. Castro, A. M. Simões, Hyers-Ulam and Hyers-Ulam-Rassias stability of a class of Hammerstein integral equations, Amer. Inst. Phys, AIP Conf. Proc. 1798(1) 020036 (2017) 10 pp.

[14] L. P. Castro, A. M. Simões, Hyers-Ulam and Hyers-Ulam-Rassias stability of a class of integral equations on finite intervals, CMMSE'17: Proceedings of the 17th International Conference on Computational and Mathematical Methods in Science and Engineering I-IV (2017) 507-515.

[15] Y. J. Cho, C. Park, T. M. Rassias, R. Saadati, Stability of Functional Equations in Banach Algebras, Springer International Publishing, Heidelberg, 2015.

[16] W.-S. Du, A generalization of Diaz-Margolis's fixed point theorem and its application to the stability of generalized Volterra integral equations, J. Inequal. Appl. 2015407 (2015) 15 pp.

[17] G. L. Forti, Hyers-Ulam stability of functional equations in several variables, Aequationes Math. 50 (1995) 143-190.

[18] Z. Gajda, On stability of additive mappings, Int. J. Math. Math. Sci. 14(3) (1991) 431-434.

[19] A. M. Hassan, E. Karapinar, H. H. Alsulami, Ulam-Hyers stability for MKC mappings via fixed point theory, J. Funct. Spaces 2016 Article ID 9623597 (2016) 11 pp.

[20] D. H. Hyers, On the stability of linear functional equation, Proc. Natl. Acad. Sci. USA 27(4) (1941) 222-224.

[21] D. H. Hyers, G. Isac, Th. M. Rassias, Stability of Functional Equations in Several Variables, Birkhäuser, Basel, 1998.

[22] S.-M. Jung, Hyers-Ulam-Rassias Stability of Functional Equations in Mathematical Analysis, Hadronic Press, Palm Harbor, 2001.

[23] S.-M. Jung, S. Şevgin, H. Şevli, On the perturbation of Volterra integro-differential equations, Appl. Math. Lett. 26 (2013) 665-669.

[24] J. R. Morales, E. M. Rojas, Hyers-Ulam and Hyers-Ulam-Rassias stability of nonlinear integral equations with delay, Int. J. Nonlinear Anal. Appl. 2 (2011) 1-6.

[25] D. Popa, I. Raşa, On the best constant in Hyers-Ulam stability of some positive linear operators, J. Math. Anal. Appl. 412(1) (2014) 103-108.

[26] Th. M. Rassias, On the stability of the linear mapping in Banach spaces, Proc. Amer. Math. Soc. 72 (1978) 297-300.

[27] Th. M. Rassias, On a modified Hyers-Ulam sequence, J. Math. Anal. Appl. 158(1) (1991) 106-113.

[28] S. Şevgina, H. Şevlib, Stability of a nonlinear Volterra integro-differential equation via a fixed point approach, J. Nonlinear Sci. Appl. 9 (2016) 200-207.

[29] C. C. Tisdell, A. Zaidi, Basic qualitative and quantitative results for solutions to nonlinear, dynamic equations on time scales with an application to economic modelling, Nonlinear Anal. 68(1) (2008) 3504-3524. 